November 2004

\title{
Complementary medicine in chronic cancer care
}

\author{
Daniel A. Monti \\ Thomas Jefferson University \\ Jingduan Yang \\ Thomas Jefferson University
}

Follow this and additional works at: https://jdc.jefferson.edu/jmbcimfp

Part of the Alternative and Complementary Medicine Commons Let us know how access to this document benefits you

\section{Recommended Citation}

Monti, Daniel A. and Yang, Jingduan, "Complementary medicine in chronic cancer care" (2004).

Marcus Institute of Integrative Health Faculty Papers. Paper 4.

https://jdc.jefferson.edu/jmbcimfp/4

This Article is brought to you for free and open access by the Jefferson Digital Commons. The Jefferson Digital Commons is a service of Thomas Jefferson University's Center for Teaching and Learning (CTL). The Commons is a showcase for Jefferson books and journals, peer-reviewed scholarly publications, unique historical collections from the University archives, and teaching tools. The Jefferson Digital Commons allows researchers and interested readers anywhere in the world to learn about and keep up to date with Jefferson scholarship. This article has been accepted for inclusion in Marcus Institute of Integrative Health Faculty Papers by an authorized administrator of the Jefferson Digital Commons. For more information, please contact: JeffersonDigitalCommons@jefferson.edu. 


\title{
Complementary Medicine in Chronic Cancer Care
}

\author{
Daniel A. Monti* and Jingduan Yang*
}

\begin{abstract}
Although advancements in cancer care have led to increased cure rates and survival times, those coping with the long-term sequelae of a cancer diagnosis often report high levels of distress, poor health-related quality of life, and unmet psychosocial needs. The shortcomings of the conventional biomedical system at sufficiently addressing these chronic illness issues are a primary reason that many patients turn to complementary and alternative medicine (CAM). Although patients usually use such treatments in addition to conventional care, they often do not discuss doing so with their conventional physicians, who are perceived as being unreceptive to the topic. Physicians may feel uninformed about CAM treatments, especially given the relatively small amount of data on the topic available in the medical literature. This article reviews a few CAM modalities commonly used by cancer survivors and those with chronic cancer illness, with a particular focus on familiarizing physicians with treatments that may potentially complement regular oncologic care by decreasing stress and enhancing health-related quality of life.
\end{abstract}

* Department of Psychiatry and Human Behavior, Jefferson Medical College, 1020 Sansom St, 1652 Thompson Bldg, Philadelphia, PA. Address reprint requests to Daniel A. Monti, MD, Section Chief of Complementary Medicine, Traditional Chinese Medicine Program, Department of Psychiatry and Human Behavior, Jefferson Medical College, 1020 Sansom St, 1652 Thompson Bldg, Philadelphia, PA. 19107. E-mail: monti1@pol net

The use of complementary and alternative medicine (CAM) treatments in the United States is substantial, especially among those with chronic medical problems, such as cancer. ${ }^{1}$ Eisenberg et al. ${ }^{2}$ published the first national survey on the use of CAM in 1993, which revealed that one in three respondents had used an unconventional or CAM treatment in the previous year. Follow-up studies have confirmed CAM use rates at least that high, ${ }^{3 \cdot 4}$ and most studies suggest that people use these treatments in addition to conventional medical care. Studies further suggest that people tend not to disclose their use of CAM treatments to their conventional physicians, with many reporting that they perceived their doctors as unreceptive to the issue. "One possible explanation for the patient-physician communication gap on this topic is the limited information most physicians have about CAM, especially given its historical absence as a covered subject in conventional Western medical training. In addition, despite a recent surge in interest in CAM from the medical community, such as the emergence of "Integrative Medicine" programs at many universities, ${ }^{5}$ there are currently limited scientific data on the topic. In response to the documented need for more research, the National Institutes of Health $(\mathrm{NIH})$ recently developed a National Center for Complementary and Alternative Medicine (NCCAM), which is charged with developing and funding CAM research.

There are thousands of CAM modalities and treatments, many of which have been used by cancer patients, either as a complement to conventional cancer treatments, or in some cases, as an alternative to conventional care. The focus of this article is the former, with an emphasis on reviewing some CAM treatments that may potentially complement standard care in cancer survivors and those with chronic cancer-related illnesses. The treatments chosen are those that have at least some supportive studies 
or encouraging preliminary data. The goal is to provide physicians who treat cancer patients with an overview of a few modalities that might be used in conjunction with standard conventional care to enhance comfort and quality of life, and to help bridge the communication gap that arises between patients and physicians when CAM is a consideration.

The three general categories of complementary modalities discussed are: (1) traditional Chinese medicine (TCM) treatments, including acupuncture and qigong; (2) mind-body medicine treatments, including meditation and hypnosis; and (3) therapeutic massage.

\section{Traditional Chinese Medicine Treatments}

\section{Theoretical Framework}

TCM is a system of health that can be traced back more than 4,000 years. It regards health as a relative balance between the individual and the outside environment. ${ }^{6}$ Specifically, everything in life is composed of the five elements-earth, wood, metal, water, and fire-and the energetic structure of all living things is a confluence of these five elements. Qi (also spelled "ch'i") refers to a type of raw energy or life force that flows in living things in characteristic patterns and channels referred to as "meridians, "which all correspond to one of the five elements. Everything in this system of health has an energetic connotation. For example, the word "liver" describes not only an anatomic entity, but also, and perhaps more importantly, an energetic center of one element (wood) that is connected with all other parts/elements of the mind-body system. Physical and emotional illness is conceptualized in terms of improper flow of qi, such as "blocked" qi through a particular meridian channel.

The state of qi along the meridians is described in terms of "yin and yang," which represent the qualitative poles of the energy circuits, such as heat and cold or stimulating and calming. A goal of TCM treatments is to achieve yin and yang balance of the meridians. A blocked meridian energy circuit will result in improper flow and an energetic imbalance towards a state of too much yin or yang. According to this system, when there is emotional overwhelm or physical distress from environmental challenges or poor lifestyle, qi can become stagnated or blocked in a given circuit, causing any number of symptoms from nausea and anxiety to pain and organ dysfunction, as well as increased susceptibility to pathogens.

\section{Acupuncture (including acupressure and electro-acupuncture)}

Acupuncture is one of the main treatment modalities of TCM. In the classic TCM model, proper use of acupuncture is based on an accurate evaluation of the meridians, which is done by palpating the pattern and characteristics of the wrist pulses and by assessing the physical manifestations of the meridians. That is, meridian imbalances are 
associated with characteristic physical symptoms; for example, dry skin corresponds to the metal element meridians; and joint pains correspond to wood element meridians. Next, the acupuncturist identifies points along the body's surface that correspond to a targeted meridian. Presumably "insertion of a fine needle at these points manipulates and rebalances the flow of qi. Alternatives to using needles include applying sustained manual pressure using a finger, called acupressure, or using electrical devices to stimulate the acupuncture points.

Whether or not the theory adequately explains the treatment effect, acupuncture has been associated with a number of health benefits to cancer patients. For example, a $\mathrm{NIH}$ Consensus Panel concluded that there is d ear evidence supporting the efficacy of acupuncture in the control of chemotherapy-related nausea and vomiting. ${ }^{7}$ More specifically, six randomized controlled clinical trials support targeting a particular point on the proximal aspect of the wrist, P6, either alone or in conjunction with stimulation at ST36 (another point on the lower leg), as an effective adjunct to standard antiemetic rnedication. ${ }^{8-13}$ These findings are consistent with a larger body of data supporting the efficacy of various methods of P6 stimulation in the control of postoperative nausea and vomiting. ${ }^{14}$ In a recent multicenter clinical trial, 739 patients treated with standard antiemetics for the relief of chemotherapy-induced nausea and vomiting were randomly assigned to either: (1) acupressure bands (which apply steady pressure to an acupuncture point on the inside of the wrist), (2) acustimulation bands (which give a mild electrical pulse to the same point), or (3) a no band control condition . Patients in the acupressure condition experienced less nausea on the day of treatment compared to controls. Among patients who received an acustimulation band, men but not women had less nausea on the day of treatment, and less nausea and vomiting overall. ${ }^{15}$

A recent review of the available studies on the use of acupuncture for chronic pain in cancer patients suggests that acupuncture might be useful in this population, although confirmatory, methodologically sound trials are lacking. ${ }^{14}$ There is, however, a randomized, single blind, placebo-controlled clinical trial that demonstrated significant pain relief in the experimental acupuncture group as compared to two types of sham acupuncture control groups, in a cohort of chronic neuropathic pain patients. ${ }^{16}$

Several studies, including one randomized controlled trial, indicate that acupuncture might be a useful method for the treatment of radiation-induced xerostomia. ${ }^{17-21}$ Acupuncture was also reported as a safe and effective treatment for some other cancerrelated conditions, such as shortness of breath caused by primary or secondary malignancy, ${ }^{22}$ lower extremity edema secondary to intrapelvic lymph node dissection for malignant gynecologic tumors, ${ }^{23}$ menopausal symptoms in women with previous breast cancer taking tamoxifen, ${ }^{24}$ and improved upper extremity mobility following axillary lymphadenectomy. ${ }^{25}$

A NIH Consensus Conference concluded that the overall frequency of acupuncturerelated adverse events is extremely low ${ }^{7}$ and prospective studies have indicated that even minor adverse events are rare. ${ }^{26}$ Acupuncture is contraindicated in the local area of an unstable spine, in persons with a severe clotting disorder, in neutropenic patients, 
and on limbs with lymphedema. ${ }^{27}$ In addition, semi-permanent needles (ie, small needles that are superficially taped to acupuncture points for days at time) should not be used in patients with valvular heart disease ${ }^{28}$; however, regular traditional acupuncture using disposable needles is not contraindicated in patients who have valvular heart disease or prosthetic valves. ${ }^{29}$

\section{Qigong}

Qigong is an ancient practice of energy manipulation through slow body movements and meditation, with or without imagery and breathing techniques. As with other modalities in TCM, qigong aims to open blocked energy channels and facilitate sufficient flow of qi. It is commonly described as "qi exercise." Even though qigong techniques can be taught in isolation for the purposes of healing and fitness, they are derived from comprehensive lifestyle systems from Buddhism and Taoism, called cultivation practices. The original cultivation practices aimed not only to improve health and fitness but also to achieve spiritual enlightenment and longevity. ${ }^{30}$ There was a huge resurgence in qigong after the Great Cultural Revolution in China during the mid-1970s. In 1999, a Chinese official made a public statement that the wide practice of falun gong (a common form of qigong) and other types of qigong was saving the country an estimated 10 billion dollars a year in medical costs. ${ }^{31}$

Despite a worldwide growing interest in qigong over the past two decades, the majority of studies on the topic have been performed in China. Chen and Yeung ${ }^{32}$ recently reviewed 50 Chinese studies on the use of qigong in cancer patients. Only a handful of these studies were considered well designed with control groups. Overall, there was some indication that patients who practiced qigong along with standard medical care showed more improvements or had better survival rates than those receiving conventional treatment alone; however, these findings cannot be considered conclusive given the methodologic limitations of the studies Outside China, a preliminary study showed that qigong practice lowered cortisol levels with concomitant changes in numbers of cytokine-secreting peripheral blood cells This study assessed 19 healthy volunteers aged 27 to 55 , before they were taught the practice of qigong and after 3,7 , and 14 weeks of daily practice. ${ }^{33}$ A randomized, controlled study that compared qigong practitioners to healthy controls found that the qigong group had significant elevations in CD4 lymphocyte count and higher CD4/CD8 lymphocyte ratio than the control group. ${ }^{34}$

Positive results from a well-designed study in patients with late-stage complex regional pain syndrome provide potential support for the consideration of qigong as a complementary intervention for management of stress-related symptoms in cancer patients. This randomized, placebo-controlled clinical trial found that qigong training was associated with short-term pain reduction and long-term anxiety reduction. ${ }^{35} \mathrm{~A}$ retrospective survey in North America also indicated a variety of physical and mental health benefits from falun gong practice, including smoking cessation, pain relief, and improved health-related quality of life. 


\section{Neuro-emotional Technique}

The neuro-emotional technique (NET) is a treatment intervention that is grounded in traditional Chinese five-element theory, but also integrates other principles with a specific focus on emotions, cognitions, and behaviors. ${ }^{1}$ NET is most applicable to cancer patients as an intervention to alleviate anxiety and traumatic stress symptoms. It has been estimated that one third of all cancer survivors suffer from traumatic stress symptoms. ${ }^{37}$ Most often, these symptoms are related to the event of receiving a cancer diagnosis or some distressing aspect of initial cancer care.

NET overlaps with standard cognitive-behavioral treatments for traumatic stress, such as exposure therapy, ${ }^{38}$ in that a major goal is to achieve a reversal (or extinction) of classically conditioned distressing emotional responses to trauma related stimuli. A major difference between NET and such treatments is that a significant component of the NET protocol involves key aspects of traditional Chinese five-element theory. The NET framework is consistent with the TCM model in that all problems, emotional or physical, involve the person's energy system. It is thought that the engagement of the energy system (by manually holding the associated meridian pulse points) in the cognitive-emotional processing of an event facilitates an expedited resolution of the event. NET is intended to be a brief, time-limited intervention. In one study, investigators demonstrated a significant decrease in phobic symptoms following a brief course (two to three visits) of a variation of NET.) ${ }^{39}$ A recently completed preliminary outcome study of NET in female cancer survivors with cancer-related traumatic stress symptoms compared pre-/post-intervention changes in response to recalling a distressing cancerrelated event. The results showed encouraging decreases in physiologic reactivity to the distressing event, decreases in subjective ratings of distress related to the event, and decreased reactivity of proinflammatory cytokines in response to recalling the event (Monti DA, Yang J, Kunkel E, et al, unpublished data, 2004).

\section{Mind-Body Techniques}

"Mind-body medicine" is a somewhat ambiguous term that generally refers to a collection of treatments that recognize the bidirectional nature of psyche and soma. Some of these modalities fall within the conventional framework, such as certain cognitive-behavioral techniques Most of the interventions discussed below are technically categorized as CAM only because they are not part of a dominant conventional therapeutic paradigm. Alleviating distress is a focus of these interventions, as it is generally recognized that stress and unmet psychosocial needs negatively affect health. ${ }^{40}$ Amplified stress in cancer patients has been associated with increased morbidity and mortality, ${ }^{41,42}$ decreased immune function, ${ }^{43}$ and increased relapse. ${ }^{44}$ Stress is thought to accumulate through a futile cycle of somatic distress generating psychological distress, which in turn advances somatic distress. ${ }^{45}$ The result is a documented increase in stress-related symptoms such as pain, anxiety, and depression that contribute to an overall decreased health outcome. ${ }^{46,47}$ The reduction of mental and physical stress-related symptoms is a primary goal in chronic illness and cancer care. ${ }^{47}$ 


\section{Hypnosis}

Franz Anton Mesmer (1734-1815) captivated the public in the 18th century when he introduced a form of hypnosis, which he called "animal magnetism."1 Mesmer made such an impact that his technique came to be known as "mesmerism," a word that is still sometimes used to describe a hypnotic-like trance. As a phenomenon, hypnosis has had a strong presence in the study of human psychology, from Jean Martin Charcot's and Pierre Janet's early work on the subject, to psychoanalysis, cognitive psychology, and brain imaging. ${ }^{48}$

The word "hypnosis" (from the Greek root hypnos, meaning sleep) is misleading in some ways because the phenomenon to which it refers is not a form of sleep; rather, it is a complex process of attentive, receptive concentration. This state, also called a "trance," is characterized by a modified sensorium, an altered psychological state and characteristically minimal motor functioning. In addition to achieving deep relaxation, the hypnotic treatment may include direct suggestions for specific changes in physiology and cognition. ${ }^{48}$ Guided imagery is often an integral pan of hypnotic technique.

There are data suggesting that hypnosis may be efficacious for a variety of mental health problems ${ }^{49,50}$ and physical disorders that are exacerbated by stress, including pain. ${ }^{51} \mathrm{~A} \mathrm{NIH}$ Technology Assessment Panel ${ }^{52}$ concluded that there was strong evidence for the use of hypnosis in alleviating chronic pain conditions, including pain associated with cancer. Hypnotic pain reduction is thought to occur through three primary mechanisms: muscle relaxation, perceptual alteration, and cognitive distraction. ${ }^{53}$ Hypnosis has been shown to be particularly helpful for a variety of acute and chronic cancer pain issues in children, ${ }^{54}$ and there is evidence to suggest that children may have better responsiveness to hypnosis than adults. ${ }^{55}$

Studies have demonstrated that hypnosis can be an effective means for some cancer patients to alleviate nausea and vomiting associated with chemotherapy ${ }_{;}^{56}$ Hypnotic techniques for this purpose focus on three major components of the problem: anxiety, conditioned responses to hospital cues, and physical responses to the chemotherapy agent. For example, the highly hypnotizable patient can frequently control nausea and vomiting by hallucinating the taste of orange or mint and dissociating from negative environmental cues. ${ }^{48}$

\section{Meditation Practice}

Many common forms of meditation are extracted from traditional Eastern systems that encompass lifestyle issues beyond the meditative techniques. For example, yoga is an ancient Eastern Indian system of health that prescribes a multiphasic approach to living, including proper diet, behavior, physical exercise, and sleep hygiene. However, it is common in the United States to practice aspects of yogic meditation without necessarily adhering to the other components of the system.

Meditation components of yoga have been integrated into a stress reduction program called mindfulness-based stress reduction (MBSR) that has demonstrated therapeutic 
benefits in several illness populations, including cancer. ${ }^{57-59}$ MBSR is a patientcentered group program that focuses on development of moment-to-moment awareness through mindfulness meditation practice in which patients learn to cultivate a capacity to be relaxed and aware in each moment. In the MBSR model, a nonjudgmental attitude is emphasized, especially with thoughts, which are never judged as wrong or faulty. In MBSR, acceptance of self in the present moment is emphasized. Mindfulness practices include sitting, walking, and body scan meditation, as well as gentle Hatha yoga.

Outcomes research in both heterogeneous and homogeneous patient populations suggest that this intervention is effective in the self-management of physical and psychological symptoms related to chronic illness. In a study of men with prostate cancer, participation in an MBSR program was reported to be a highly beneficial means of coping. ${ }^{60}$ Likewise, a controlled study of a mixed group of cancer patients reported significant improvements in mood disturbances and decreased stress: these improvements were maintained at 6-month follow-up ${ }^{61}$ One study found that the mood improvement associated with MBSR correlated to left-sided anterior activation, which is thought to be due to an increase in positive affect. ${ }^{62}$ Another study reported that breast and prostate cancer patients who received the 8-week MBSR program had shifts in their immune profiles (reduction in Th1 proinflammatory lymphocyte to Th2 anti-inflammatory lymphocyte ratio), which correlated with decreased depressive symptoms. ${ }^{63} \mathrm{~A}$ study of breast cancer patients showed that participants who received the MBSR program reported greater feelings of refreshment post -sleep than other panicipants. ${ }^{64}$

Mindfulness-based an therapy (MBAT) is a newly developed program that integrates MBSR within a supportive expressive group formal then was designed specifically for cancer patients. ${ }^{65,66}$ A recent randomized, controlled trial of MBAT showed significant reductions in psychological distress $(P<.0001)$ and improvements in quality of life $(P=$ .001 ) in a group of women with mixed cancer diagnoses as compared to wait-list controls (Monti DA, Peterson C Kunkel E, et al, unpublished data, 2004). MBAT uses the core components of the standard MBSR program. However, MBAT differs from MBSR in that (1) MBAT is designed specifically for cancer patients, whereas MBSR is intended for a wide range of populations; (2) MBAT provides a nonverbal creativeexpressive component by means of guided constructive tasks with various art media (an therapy); and (3) MBAT incorporates the mindfulness practices within a supportive expressive group environment that is intended for seven to 10 participants, similar to traditional group psychotherapy. In contrast, MBSR groups do not emphasize group interaction, thus allowing for 30 or more participants in a given group.

\section{Therapeutic Massage}

Massage is defined as the systematic manual or mechanical manipulations of the soft tissues of the body by such movements as rubbing, kneading, pressing, rolling, and slapping for therapeutic purposes such as promoting circulation of the blood and lymph, relaxation of muscles, relief of pain, and other physical and mental benefits.66 The practice of massage dates back thousands of years in the ancient cultures of China, Japan, and India, spreading to Europe some time around 300 BC. 
The literature suggests three potential therapeutic benefits of massage: relaxation, decreased pain, and improved sleep. Relaxation has been studied in terms of physiologic indicators that include decreased heart rate, blood pressure, respiratory rate, and electromyographic activity. Studies also have considered levels of cortisol or catecholamines, increased skin temperature, and galvanic skin responses. A recent review identified 10 studies that investigated relaxation parameters, seven of which found significant changes in the expected direction in one or more of the physiologic indicators mentioned above.68 Findings overall suggested a correlation between massage therapy and decreased cortisol levels, decreased catecholamines, and decreased anxiety.

There are several references in the literature supporting the use of massage for pain relief, including three reports that specifically studied cancer patients. One study of 28 hospitalized patients with cancer pain compared a 10-minute Swedish back massage to a 10-minute attention-control intervention, and found decreased pain levels in the massage group for male, but not female, participants. ${ }^{69}$ In a small, randomized, controlled clinical trial of men with cancer, a massage intervention group was associated with increased relaxation and significant pain relief as compared to two nonintervention control groups. ${ }^{70}$ A study of nine male hospitalized cancer patients showed a large decrease in pain levels following two 30-minute sessions of massage to feet, back, neck, and shoulders.

It is possible that massage contributes to decreased pain and increased relaxation, which could lead to improved sleep, which reciprocally could contribute to decreased pain and increased relaxation. Adequate sleep is important for physical and psychological health. Sleep deprivation has been associated with increased psychiatric symptoms, increased pain, and decreased quality of life. ${ }^{71}$ There are three reports in the literature on the potential effects of massage on sleep, although non of these studies included cancer patients.

One study examined the effects of a 30-minute back massage for 5 consecutive days in pediatric psychiatric inpatients. ${ }^{72}$ The results suggested that those in the massage group reported fewer psychiatric symptoms and spent a significantly greater amount of time sleeping in bed at night. A study of chronic fatigue syndrome patients compared the effects of massage therapy and transcutaneous electrical stimulation. The massage group demonstrated more hours or sleep, fewer somatic and psychiatric complaints, and lower urinary cortisol levels. ${ }^{73}$ A study of critically ill men suggested sleep improvement from brief daily back massages as compared 10 mental imagery and nonintervention control, however, statistical issues make this study inconclusive. ${ }^{74}$

\section{Conclusion}

Although the effectiveness of most CAM treatments has not been examined extensively, there is some support for the use of several modalities as 
potential complements to conventional cancer care, particularly for the qualityof-life issues associated with a chronic illness. .For example pain is a major problem for many cancer patients. There is much support for the use of hypnosis in managing pain associated with medical procedures and some support for its use in managing chrome cancer pain. There is less evidence to support the use of TCM modalities such as acupuncture. as well as meditation and massage for management of chronic cancer pain. On the other hand, there are some compelling data to support the use of acupuncture for nausea and vomiting associated with cancer treatments, and there are some good preliminary data suggesting that qigong, NET, meditation techniques, and massage can decrease distress levels and improve sense of well-being.

Cancer patients use a wide variety of nonpharmacologic treatments regardless of effectiveness, which means clinicians should be aware of the common modalities for which there is available research. Several studies have demonstrated a lack of awareness of nonpharmacologic strategies in general among healthcare professionals, which suggests there is much to learn about which treatments are effective and which are not. ${ }^{51}$ The physician-patient relationship would benefit from the physician being able to discuss specific complementary treatments with patients, rather than avoid the topic or dismiss such treatments as elusive or not part of conventional cancer care.

\section{References}

1 Monti DM, Stoner M: Complementary and Alternative Medicine, in Kornstein SG, Clayton AH (eds): Women's Mental Health. New York, NY, Guilford. 2002, pp 344356

2 Eisenberg DM, Kessler RC, Foster C, et al: Unconventional medicine in the United States: Prevalence, costs and patterns of use. N Engl J Med 328:246-252, 1993

3 Elder N, Gillcrist A. Minz R: Use of alternative health care by family practice patients. Arch Fam Med 6 181-184, 1997

4 Eisenberg DM, Davis RB, Ettner SL, et al: Trends in alternative medicine use in the United States, 1990-1997: Results of a follow-up national survey. JAMA 280:15691575, 1998

5 Wetzel MS, Eisenberg DM, Kaptchuk TJ: Courses involving complementary and alternative medicine at US medical schools. JAMA 280: 784-787,1998

6 Beal MW: Acupuncture and acupressure: Applications to women's reproductive health care. J Nurse Midwife 44:217-230, 1999

7 NIH Consensus Conference. Acupuncture. JAMA 280:1518-1524, 1998

8 Dundee JW, Ghaly RG, Fitzpatrick KT, et al: Acupuncture to prevent cisplatinassociated vomiting. Lancet 1:1083-1083, 1987 (letter)

9 Shen J, Wenget NS, Glaspy JA, et al: Adjunct antiemesis electroacupuncture m stem cell transplantation. Proc Am Soc Clin Oncol 16: A148, 1997 (abstr) 
10 Price $\mathrm{H}$, Lewith $\mathrm{G}$, Williams C: Acupressure as an antiemetic in cancer chemotherapy. Comp Med Res 5:93-94, 1991

11 McMillan C, Dundee JW, Abram WP: Enhancement of the antiemetic action of ondansetron by transcutaneous electrical stimulation of the P6 antiemetic point. In patients having highly emetic cytotoxic drugs. Br J Cancer 64:971-972, 1991

12 Shen J, Wenger N, Glaspy J, et al: Electroacupuncture for control of myeloablative chemotherapy-induced emesis. A randomized controlled trial. JAMA 284:27552761, 2000

13 Pearl ML, Fischer M, McCauley DL, et al: Transcutaneous electrical nerve stimulation as an adjunct for controlling chemotherapy-induced nausea and vomiting in gynecologic oncology patients. Cancer Nurs 22 307-311, 1999

14 Weiger WA, Smith M. Boon H, et al: Advising patients who seek complementary and alternative medical therapies for cancer. Ann Intern Med 137 889-903,2002

15 Roscoe JA, Morrow GR, Hickok JT, et al: The efficacy of acupressure and acustimulation wrist bands [or the relief of chemotherapy-induced nausea and vomiting: A University of Rochester Cancer Center Community Clinical Oncology Program multicenter study. J Pain Symptom Manage 26:731-742, 2003

16. Ahmi D, Rubino C, Pichard-Leandri E, et al: Analgesic effect of auricular acupuncture for cancer pain: A randomized, blinded, controlled trial. J Clin Oncol 21.4120-4126, 2003

17. Blom M, Dawidson I, Fernberg JO, et al: Acupuncture treatment of patients with radiation-induced xerostomia. Eur J Cancer B Oral Oncol 32B:182-189, 1996

18. Blom M, Lundeberg T: Long-term follow-up of patients treated with acupuncture for xerostomia and the influence of additional treatment. Oral Dis 6:15-24, 2000

19. Davidson I, Angmar-Mansson B, Blom M, et al: Sensory stimulation (acupuncture) increases the release of calcitonin gene-related peptide in the saliva of xerostomia sufferers. Neuropeptides 33:244-250, 1999

20. Johnstone PA, Niemtzow RC, Riffenburgh RH, et al: Acupuncture for xerostomia: Clinical update. Cancer 94:1151-1156, 2002

21. Rydholm M, Strang P: Acupuncture for patients in hospital-based home care suffering from xerostomia. J Pallial Care15:20-23, 1999

22. Filshie J, Penn K, Ashley S: Acupuncture for the relief of cancer -related breathlessness. Palliat Med 10:145-150, 1996

23. Kanakura Y, Niwa K, Kometani K: Effectiveness of acupuncture and moxibustion treatment for lymphedema following intrapelvic lymph node dissection: A preliminary report. Am J Chin Med 30:37-43, 2002

24. Porzio G, Trapasso T, Martelli S: Acupuncture in the treatment of menopauserelated symptoms in women taking tamoxifen. Tumori 88: 128-130, 2002

25. He JP, Friedrich M, Ertan, AK, et al: Pain-relief and movement improvement by acupuncture after ablation and axillary lymphadenectomy in patients with mammary cancer. Clin Exp Obstet Gynecol 26 81-84, 1999

26. Kaptchuk TJ: Acupuncture:Theory, efficacy, and practice. Ann Intern Med 1363 74383, 2002

27. Mortomer PS, Badger C, Hall JG: Lymphedema, in Doyle D, Hanks GWC, MacDonald N (eds): Oxford Textbook of Palliative Medicine. Oxford, UK, Oxford University Press, 1998, pp 657-665 
28. Filshie J: Safety aspects of acupuncture in palliative care. Acupunct Med 19:117122, 2001

29. Stellon A: Acupuncture in patients with valvular heart disease and prosthetic valves. Acupunct Med 21:87-91, 2003

30. Li HZ: Zhuan Falun. Taibei, China, YiQuan, 2003

31. Fang B: An opiate of the masses? Millions of Chinese embrace a mystical exercise movement. US News and World Report, February 22, 1999

32. Chen K, Yeung R: Exploratory studies of qigong therapy for cancer in China. Integr Cancer Ther 1:345-370, 2002

33. Jones BM: Changes in cytokine production in healthy subjects practicing Guolin qigong: A pilot study. BMC Complement Altern Med 1:811, 2001

34. Lee MS, Huh HJ, Jeong SM, et al: Effects of qigong on immune cells. Am J Chin Med 31:327-335, 2003

35. WU WH, Bandilla E, Ciccone DS, et al: Effects of Qigong on late-stage complex regional pain syndrome. Altern Ther Health Med 5:45-54, 1999

36. Yang J, Monti D, Patkar A, et al: Effects of falun gong on physical and mental health. Presented at the American Psychiatric Association Annual Meeting, Philadelphia, PA, May 18, 2002

37. McGarvey EL: Evidence of acute stress after diagnosis of cancer. South Med J 91:864-866, 1998

38. Faa EB, Jaycock LH: Cognitive-behavioral theory and treatment of post-traumatic stress disorder, in Spiegel D (ed): Efficacy and Cost Effectiveness of Psychotherapy. Washington, DC, American Psychiatric Press, 1999, pp 23-61

39. Peterson KB: The effects of spinal manipulation on the intensity of emotional arousal in phobic exposed to threat stimulus: A randomized. controlled, doubleblind clinical trial. J Manipulat Physiol Ther 20: 602-606, 1997

40. Cohen M, Kunkel E, Levenson J: Associations between psychological stress and malignancy, in Hubbard J, Workman E(eds): Handbook of Stress Medicine: An Organ System Approach. Boca Raton, FL, CRC Press, 1998, pp 205-228

41. Spiegel D, Bloom J, Yalom I: Group support for patients with metastatic breast cancer: A randomized prospective outcome study. Arch Gen Psychiatry 38:527533,1981

42. Ell K, Nishimoto R, Mediansky J, et al: Socia! relations, social support and survival among patients with cancer. Psychosom Res 36:531-541, 1992

43. Fawzy I, Fawzy N, Hyun C, et al: Malignant melanoma: Effects of an early structured psychiatric intervention, coping and affective state on recurrence and survival 6 years later. Arch Gen Psychiatry 50:681-689, 1993

44. Uchino B, Cacioppo J, Kiecoult-Glaser J: The relationship between social support and physiological processes: A review with emphasis on underlying mechanisms and Implications for health. Psychol Bull 119 488-531, 1996

45. Jacobsen P, Holland J: The stress of cancer - Psychological responses to diagnosis and treatment, in Cooper C, Watson M(eds): Cancer and Stress -Psychological, Biological, and Coping Mechanisms, Chichester, UK, Wiley, 1991, pp 147-169

46. Pasacreta J, Pickett M: Psychosocial aspects of palliative care. Semin Oncol Nurs 14:110-120, 1994 
47. Kunkel E, Bakker J, Myers R, et al: Biopsychosocial aspects of prostate cancer. Psychosomatics 41:85-94, 2000

48. Spiegel H, Greenleaf M, Speigel D: Hypnosis, in Saddock BJ, Saddock VA(eds): Kaplan and Saddock's Comprehensive Textbook of Psychiatry. Philadelphia, PA, Lippincott Williams \&: Wilkins, 2000, pp 2128-2145

49. Marks IM, Gelder MG, Edwards G: Hypnosis and desensitization for phobics: A controlled prospective trial. Br J Psychiatry 114:1263-1274, 1968

50. Stanton HE: Using hypnotherapy to overcome examination anxiety. Am J Clin Hypn 35:198-204, 1993

51. Sellick SM, Zaza C: Critical review of 5 non-pharmacologic strategies for managing cancer pain. Cancer Prev Control 2:7-14,1998

52. National Institutes of Health (NIH): Technology assessment panel on integration of behavioral and relaxation approaches into the treatment of chronic pain and insomnia. NIH Meeting, Bethesda, MD, 1996

53. Spiegel H, Speigel D: Trance and Treatment: Clinical Uses of Hypnosis. New York, NY, Basic Books, 1978

54. Liossi C, Haura P: Clinical hypnosis in the alleviation of procedure-related pain in pediatric oncology patients. Int J Exp Hypn 51:4-28, 2003

55. Wakeman RJ, Kaplan JZ: An experimental study of hypnosis in painful burns. Am J Clin Hypn 21:3-12,1978

56. Genuis ML: The use of hypnosis in helping cancer patients control anxiety, pain, and emesis: A review of empirical studies. Am J Clin Hypn 37:316-325, 1995

57. Kabat-Zinn J, Massion AO, Kristeller J, et al: Effectiveness of a meditation-based stress reduction program in the treatment of anxiety disorders. Am J Psychiatry 149:936-943, 1992

58. Kabat-Zinn J, Lipworth L, Burney R, et al: Four-year follow-up of a meditationbased program for self-regulation of chronic pain: Treatment outcomes and compliance. Clin J Pain 2:159-173,1987

59. Reibel D, Greeson J, Brainard G: Mindfulness-based stress reduction and healthrelated quality of life in a heterogeneous patient population. Gen Hosp Psychiatry 23:183-192, 2001

60. Coker KH: Meditation and prostate cancer. Semin Urol Oncol 17:111-118, 1999

61. Carlson L, Ursuliak Z, Goodey E, et al: The effects of mindfulness-based stress reduction program on mood and symptoms of stress in cancer patients: 6-month follow-up. Support Care Cancer 9:112 -113, 2001

62. Davidson RJ, Kabat-Zinn J: Alterations in brain and immune function produced by mindfulness meditation. Psychosorn Med 65:564-570, 2003

63. Carlson LE, Speca M: Mindfulness-based stress reduction in relation to quality of life, mood, symptoms of stress, and Immune parameters 111 breast and prostate cancer out subjects. Psychosom Med 65:571-581, 2003

64. Shapiro SL, Lopez SF, Schwartz GE, et al: Quality of life and breast cancer: Relationship to psychosocial variables. J Clin Psychol 57:501-519, 2001

65. Schacht MJ, Monti DA, Peterson C, et al: Integrative tumor board: Recently diagnosed prostate cancer. Integr Cancer Ther 2:81-83, 2003

66. Monti DA, Peterson C: Mindfulness-based art therapy: Results from a two-year study. Psychiatr TImes 21:63-66, 2004 
67. Beck MF: Miladay's Theory and Practice of Therapeutic Massage. Albany, NY, Miladay, 1999

68. Field T: Massage therapy effects. Am Psychol 53:1270-1281, 1998

69. Weinrich SP, Weinrich MC: The effect of massage on pain in cancer patients. Nurs Res 3:J40-J45, 1990

70. Wilke DJ, Karnpbell J, Cutshall S, et al: Effects of massage on pain intensity, analgesics and quality of life in patients with cancer pain. A pilot study of a randomized clinical trial conducted within hospice care delivery. Hospice J 15:3153,2000

71. Sunshine W, Field TM, Quintino O, et al: Fibromyalgia, benefits from massage therapy and transcutaneous electrical simulation. J Clin Rheum 218-22, 1996

72. Field T, Morrno C, Valdeon C, et al: Massage reduces anxiety in child and adolescent psychiatric patients. J Am Acad Adolesc Psychiatry 31:125-131,1992

73. Field T, Sunshine W, Hernandez-Riel M, et al: Massage therapy effects of depression and somatic symptoms in chronic fatigue. J Chronic Fatigue Syndr 3:43-51, 1997

74. Richards KC: The effects of back massage and relaxation intervention on sleep in critically ill patients. Am J Crit Care 7:288-299, 1998 